\title{
Cycled enteral antibiotics in patients on long-term parenteral nutrition and the incidence of systemic sepsis
}

\author{
R. Dobson ${ }^{1}$, A. R Barclay ${ }^{2}$, C. McGukin ${ }^{1}$, G. Walker ${ }^{1}$, P. McGrogan ${ }^{1}$ and D. Flynn ${ }^{1}$ \\ ${ }^{1}$ Department of Paediatric Gastroenterology and Nutrition, Royal Hospital for Sick Children, Dalnair Street, \\ Glasgow G3 8SJ, UK and ${ }^{2}$ Division of Developmental Medicine, University of Glasgow, Glasgow G12 8QQ, UK
}

Parenteral nutrition (PN) has been accepted as supportive therapy for patients with intestinal failure, either until bowel adaptation can occur or until definitive treatment (intestinal transplantation) can be given in irreversible disease. However, it is associated with considerable morbidity and mortality and the most important complication is that of PN-related sepsis ${ }^{(1,2)}$. The exact mechanisms of sepsis in these patients remain controversial. However, many episodes may relate to translocation of bacteria through the wall of the underused intestine $^{(3)}$. Preventative strategies proposed for bacterial translocation include the use of 'cycled' enteral antibiotics. This strategy comprises a defined regimen of oral antibiotics cycling between different preparations and periods of cessation, normally for 1-2 weeks at a time. Commonly-used regimens include colistin and tobramycin alternating with metronidazole. The antibiotics themselves help to reduce bacterial overgrowth, which is believed to increase the likelihood of translocation, and the cyclical use is believed to reduce the development of bacterial resistance to antibiotic therapy ${ }^{(4,5)}$. Case notes of eight patients on long-term PN were reviewed and the incidence of episodes of sepsis before and after the introduction of cycled enteral antibiotics was calculated. The Wilcoxon signed rank test was used to establish whether or not the observed difference in incidence of sepsis was significant.

\begin{tabular}{lcc}
\hline & \multicolumn{2}{c}{ Total sepsis rate } \\
\cline { 2 - 3 } Patient & $\begin{array}{c}\text { Before starting } \\
\text { antibiotics }\end{array}$ & $\begin{array}{c}\text { After starting } \\
\text { antibiotics }\end{array}$ \\
\hline 1 & 1.62 & 1.43 \\
2 & 1.72 & 1.59 \\
3 & 2.39 & 0.84 \\
4 & 2.85 & 0.55 \\
5 & 3.17 & 0.56 \\
6 & 1.31 & 1.06 \\
7 & 1.88 & 1.06 \\
8 & 3.68 & 2.89 \\
Mean & 2.33 & 1.25 \\
Median & 3.10 & 1.06 \\
SD & 0.83 & 0.76 \\
\hline Wilcoxon statistic $-252 ; P=0.012$ &
\end{tabular}

Wilcoxon statistic $-2.52 ; P=0.012$.

There was a significant reduction in the number of episodes of systemic sepsis once cycled enteral antibiotics were introduced, presumably due to the inhibitory effect of the antibiotics on translocation of bacteria from the intestinal lumen. However, the sample size of the present study was small and the patients differed considerably in terms of age, diagnosis, co-morbidity and proportion of total nutrition derived from enteral rather than PN. Ultimately, more studies with larger and more standardised population samples are required. However, the results of the present study are certainly suggestive of a benefit for most patients started on enteral antibiotics. In patients who have already suffered a number of septic episodes whilst on total PN, it may well be appropriate to at least trial rotational enteral antibiotics in order to attempt to reduce the number of septic episodes suffered.

1. Forbes A (2007) Curr Opinion Gastroenterol 23, 183-186.

2. Torres C \& Vanderhoof J (2006) Curr Paediat 16, 291-297.

3. MacFie J, Reddy B \& Gatt M (2006) Br J Surg 93, 87-93.

4. Quigley E \& Quera R (2006) Gastroenterology 130, S78-S90.

5. Vanderhoof J, Young R, Murray N et al. (1998) J Pediatr Gastroenterol Nutr 27, 155-160. 